\title{
RNA-guided genome editing à la carte
}

\author{
Cell Research (2013) 23:733-734. doi:10.1038/cr.2013.39; published online 12 March 2013
}

Two recent papers in Science illustrate how the prokaryotic CRISPR-Cas immune system machinery, which typically targets invasive genetic elements such as viruses and plasmids, can be converted into a sophisticated molecular tool for next-generation human genome editing. The versatile Cas9 RNA-guided endonuclease can be readily reprogrammed using customizable small RNAs for sequence-specific single- or double-stranded DNA cleavage.

Molecular biologists can now order restriction enzymes à la carte, to target any DNA template and generate cleavage at any chosen site precisely, without the agony of finding the right cutter, or the caveat to settle for the nearest acceptable site. Even better, the chef also offers the choice of double-stranded DNA blunt cleavage, or strand-specific nicking. Indeed, two recent milestone reports in Science [1,2] describe the diversion of the CRISPR-Cas bacterial adaptive immune system in novel genome editing applications in mammalian cells.

In bacteria and archaea, CRISPR (clustered regularly interspaced short palindromic repeats) and CRISPR-associated proteins (Cas) naturally provide adaptive immunity against viruses and plasmids through the uptake and stockpiling of small fragments (sequences of about $30 \mathrm{bp}$ named "spacers") of the encountered genetic elements into CRISPR arrays, in between direct repeats [3]. The subsequent transcription of a CRISPR array into a precursor RNA (pre-crRNA), followed by a maturation step in which the pre-crRNA is diced into smaller CRISPR RNAs (crRNAs), produces a cocktail of small interfering RNAs that are loaded onto the patrolling Cas-encoded machinery - involving helicase and nuclease activities - to specifically target and destroy nucleic acid showing sequence complementarity to the spacers [4]. Although generic, this scheme shows marked idiosyncrasies when considering the numerous and highly diverse CRISPR-Cas systems that have been examined [5]. For instance, the thoroughly studied Type II systems require an additional, small non-coding RNA called trans-activating CRISPR RNA (tracrRNA) to achieve the pre-crRNA maturation step (Figure 1A) [6]. To date, CRISPR-Cas systems have been used to enhance resistance against viruses in bacteria of industrial interest, and studies have shown that bacteria can be vaccinated to preclude the uptake (and dissemination) of antibiotic resistance genes [7]. The hypervariable nature of these rapidly evolving loci has also been used for phylogenetic and evolutionary studies.

Until recently, the most advanced genome editing technologies involved three families of site-specific nucleases, namely zinc finger nucleases (ZFNs), transcription activator-like effector nucleases (TALENs), and the engineered homing meganucleases [8]. These technologies can trigger errorprone sequence repair at the cleavage site through either non-homologous end joining (NHEJ) or homology directed repair (HDR) pathways, generating genetically "edited" variants. However, despite successful uses in various eukaryotic hosts and model systems, they all present important limitations that still warrant further developments in this field. Notably, off-target mutagenesis activity due to imperfect specificity in target sequence recognition may explain certain cases of nuclease toxicity within transfected cells, but above all is the significant time and cost requirement for (re)developing such engineered, customized proteins for each desired target sequence.

A step-change in genome editing perspectives occurred in 2012 when Jinek et al. [9] reported that the streptococcal Cas9 endonuclease can drive sequence-specific DNA cleavage in the presence of a synthetic, hybrid RNA molecule mimicking both crRNA and tracrRNA. Site-directed mutagenesis of Cas9 in either the RuvC- or HNH-motif showed strand cleavage specificity, thereby providing two strand-specific nickases, in addition to the wild-type endonuclease $[9,10]$.

Recently, Cong et al. [1] and Mali et al. [2] showed that Type II CRISPRCas systems can be adapted to and used in mouse and human cell lines, to specifically induce mutations and edit genetic loci of interest. Mali et al. [2] engineered the Streptococcus pyogenes Cas9 nuclease using fusions of crRNA and tracrRNA to target the AAVS1 locus in human 293T cells, K562 cells, and induced pluripotent stem (iPS) cells, with targeting rates competitive with TALENs and ZFNs. The system relies on a human codon-optimized Cas9 nuclease bearing a SV40 nuclear localization signal, in combination with a guide RNA that targets a $\mathrm{GN}_{21} \mathrm{GG}$ sequence (to ensure human U6 polymerase III promoter and CRISPR targeting requirements) (Figure 1B). They also simultaneously used guide RNAs 
A

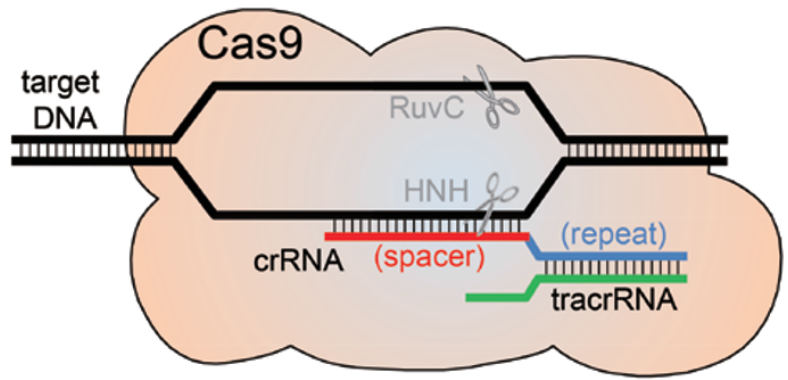

B

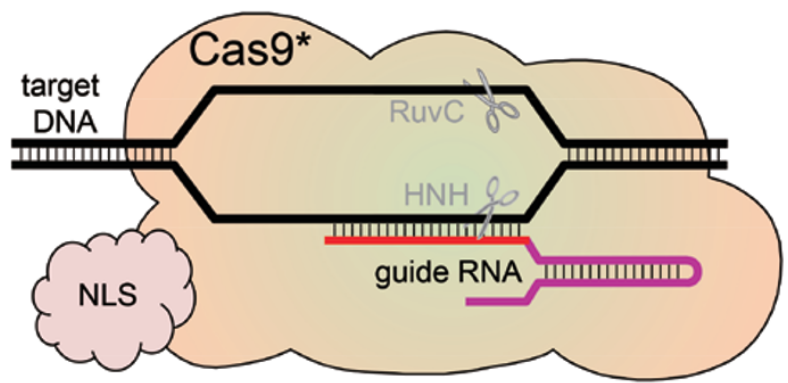

Figure 1 Model for RNA-guided DNA cleavage by Cas9. (A) Native system. In Type II CRISPR-Cas systems, sequence complementarity between the crRNA (in its spacer-derived moiety) and the target genome leads to an R-loop structure, where cleavage of each DNA strand is driven by a specific domain (HNH or RuvC) of the Cas9 endonuclease. The trans-activating CRISPR RNA (tracrRNA) needs to base pair with the precursor crRNA (in its repeat-derived moiety) in order to generate mature crRNA. (B) Engineered system. The Cas9 nuclease may be modified by codon optimization for increased expression within the heterologous host, fusion to a nuclear localization signal (NLS) for proper compartmentalization, and sitedirected mutagenesis of either the $\mathrm{HNH}$ or RuvC domain to convert the nuclease into a strand-specific nickase. Specificity of the cleavage within the target genome is determined by the spacer-like moiety (in red) of a chimeric guide RNA molecule, which mimicks the native crRNA:tracrRNA complex.

to target multiple loci concurrently. In silico analyses predict that this versatile system could target an impressive $40 \%$ of exons in the human genome, illustrating the broad application scope of this technology. Concomitantly, Cong et al. [1] engineered the Streptococcus pyogenes and Streptococcus thermophilus Type II CRISPR-Cas systems to show that Cas9 nucleases can introduce double-stranded breaks and induce precise genome editing in human and mouse cells. They showed that the native Cas9:RNAseIII:tracrRNA:crRNA system can target EMX1 in human cells. They also showed that a chimeric crRNA-tracrRNA hybrid in combination with Cas9 can target $E M X 1$ and $P V A L B$ in human cells and $T h$ in mouse cells, with cleavage efficiency matching TALENs. Further, they likewise provided a multiplexing proof of concept by simultaneously editing $E M X 1$ and PVALB sites using a single CRISPR array containing two distinct spacers. The ability to (re)direct the Cas9 endonuclease with several guide RNAs that target various sequences opens new avenues for concurrent editing of multiple genomic targets.

Overall, this versatile system provides unprecedented flexibility with customized "reprogrammable" RNA guides associated with high-fidelity specific Cas endonucleases, paving the way for next-generation genome editing. The chef has opened the menu for our voracious scientific appétit with these delectable molecular machines.

Philippe Horvath ${ }^{1}$, Rodolphe Barrangou ${ }^{2,3}$

${ }^{1}$ DuPont Nutrition and Health, Dangé-SaintRomain, 86220, France; ${ }^{2}$ DuPont Nutrition and Health, Madison, WI 53716, USA; ${ }^{3} \mathrm{New}$ address: Department of Food, Bioprocessing and Nutrition Sciences, North Carolina State University, Raleigh, NC 27695, USA Correspondence: Philippe Horvath Tel: +33-549-191-209; Fax: +33-549-864-839 E-mail: philippe.horvath@dupont.com

\section{References}

1 Cong L, Ran FA, Cox D, et al. Science 2013; 339:819-823.

2 Mali P, Yang L, Esvelt KM, et al. Science 2013; 339:823-826.

3 Barrangou R, Fremaux C, Deveau $\mathrm{H}$, et al. Science 2007; 315:1709-1712.

4 Horvath P, Barrangou R. Science 2010; 327:167-170.

5 Makarova KS, Haft DH, Barrangou R, et al. Nat Rev Microbiol 2011; 9:467-477.

6 Deltcheva E, Chylinski K, Sharma CM, et al. Nature 2011; 471:602-607.

7 Garneau JE, Dupuis ME, Villion M, et al. Nature 2010; 468:67-71.

8 Perez-Pinera P, Ousterout DG, Gersbach CA. Curr Opin Chem Biol 2012; 16:268277.

9 Jinek M, Chylinski K, Fonfare I, et al. Science 2012; 337:816-821.

10 Gasiunas G, Barrangou R, Horvath P, et al. Proc Natl Acad Sci USA 2012; 109:E2579E2586. 